\title{
Changing Pattern of Paediatric Anaphylaxis in Hong Kong, 2010-2019
}

\author{
Agnes Sze Yin Leung ${ }^{1}$, Rebecca Ming Yan $\mathrm{Li}^{2}$, Ann Wing Shan $\mathrm{Au}^{2}$, Jaime Sou da Rosa \\ Duque $^{3}$, Po Ki Ho ${ }^{4}$, Gilbert Chua ${ }^{5}$, Chi Hang Wong ${ }^{6}$, David Chi Kong Luk ${ }^{6}$, Mike Yat \\ Wah Kwan ${ }^{7}$, Yat Sun Yau ${ }^{4}$, Gary Wong (N/A)로 ${ }^{1}$ and Ting Fan Leung ${ }^{1}$ \\ ${ }^{1}$ The Chinese University of Hong Kong \\ ${ }^{2}$ The Chinese University of Hong Kong Faculty of Medicine \\ ${ }^{3}$ University of Hong Kong Li Ka Shing Faculty of Medicine Department of Paediatrics and \\ Adolescent Medicine \\ ${ }^{4}$ Hospital Authority Kowloon Central Cluster \\ ${ }^{5}$ University of Hong Kong Li Ka Shing Faculty of Medicine \\ ${ }^{6}$ Hospital Authority \\ ${ }^{7}$ Hospital Authority Kowloon West Cluster
}

July 1, 2021

\begin{abstract}
Background: Anaphylaxis is a significant health burden in most Western countries but there is little published data on the incidence and pattern of anaphylaxis in Asia. We aim to determine the incidence rate and pattern of anaphylaxis over the past decade among the paediatric population in Hong Kong. Methods: Medical records of patients presenting with allergy-related symptoms during the period 2010 to 2019 were examined. Paediatric patients aged below 18 years who fulfilled the diagnostic criteria for anaphylaxis laid out by the NIAID/FAAN were analysed. Incidence rates were calculated using population statistics as the denominator. All information pertaining to the anaphylaxis events and patients' characteristics were retrieved using standardized data collection forms. Results: The overall 10-year estimated incidence of anaphylaxis was 7.70 per 100,000 person-years, with a rising trend of anaphylaxis incidence across time. Food-induced anaphylaxis accounted for the majority of hospital presentation, of which peanut and shellfish were the top food triggers in our population. Comorbid asthma and young age were risk factors associated with wheeze at presentation. Misdiagnosis of anaphylaxis occurred in up to half the anaphylaxis cases and adrenaline was only utilised in $45 \%$ of cases. Conclusions: An increasing trend of anaphylaxis incidence over the past decade is evident in Hong Kong children, with a discrepantly low accuracy in diagnosis and suboptimal management of anaphylaxis. There is a pressing need to heighten public and physicians' awareness of the distinctive features of anaphylaxis in the paediatric age group.
\end{abstract}

\section{Hosted file}

Changing Pattern of Paediatric Anaphylaxis in HK.PAI_21Jun21.docx available at https: //authorea.com/users/344824/articles/528599-changing-pattern-of-paediatric-anaphylaxisin-hong-kong-2010-2019 